\title{
Building a National Simulation Program in Rwanda Through the Use of Partnerships
}

\author{
Philomene Uwimana ${ }^{1}$, Renee Pyburn ${ }^{1}$ \\ ${ }^{1}$ University of Rwanda, College of Medicine and Health Sciences, Rwanda
}

\section{Background}

Building a simulation program in a low-resource environment can be challenging. With the assistance of international partners, Rwanda has successfully built a sustainable national simulation program. We believe this may be a successful model for other low-resource countries.

\section{Description}

In 2003, a partnership was formed between Kigali Health Institute (KHI) and VVOB (the Flemish International Corporation in Belgium). Through this partnership former KHI, currently College of Medicine and Health Sciences of the University of Rwanda - ( UR-CMHS) was able to build an initial simulation program including an education plan, outfitting rooms, training standardized patients, purchasing and the setting up of equipment, and training simulation staff and faculty. This partnership was extended until 2010. From 2010 to 2012, a partnership was formed between the Kigali University Teaching Hospital (CHUK) and the Canadian Anesthesiologists Society International Education Foundation. Through this partnership a skills lab was set up at CHUK, for use by medical students, residents, and physicians at CHUK. From 2012-2015, a third simulation partnership was set up with the Human Resources for Health Program whereby an experienced simulation advisor was sent to CMHS for one year. A number of improvements have been accomplished through this partnership.

\section{Lessons Learned}

Through the use of deliberate, structured international partnerships, a successful and sustainable simulation program has been set up in Rwanda. We have learned that the partnerships should be designed in a way that ensures sustainable growth and improvement in the simulation program long after partnerships terminate.

\section{Conclusion}

International simulation program partnerships, if set up correctly, can assist in building sustainable simulation facilities in low-resource settings.

Key words: simulation, clinical education, low resource countries, Rwanda, partnerships 